\title{
Research Article \\ Convection-Radiation Heat Transfer in a Nonlinear Fluid with Temperature-Dependent Viscosity
}

\author{
Mehrdad Massoudi, ${ }^{1}$ Phuoc X. Tran, ${ }^{1}$ and R. Wulandana ${ }^{2}$ \\ ${ }^{1}$ U.S. Department of Energy, National Energy Technology Laboratory (NETL), P.O. Box 10940, \\ Pittsburgh, PA 15236, USA \\ ${ }^{2}$ Department of Mechanical Engineering and Materials Science, University of Pittsburgh, \\ Pittsburgh, PA 15261, USA \\ Correspondence should be addressed to Mehrdad Massoudi, mehrdad.massoudi@netl.doe.gov
}

Received 25 February 2009; Accepted 7 June 2009

Recommended by Kumbakonam R. Rajagopal

We will study the fully developed flow of a generalized second-grade fluid down an inclined plane. The viscosity is not only a function of the shear rate (shear-thinning or shear-thickening) but also depends on the temperature in an exponential way. The effects of radiation boundary condition at the free surface are considered. The boundary value problem is solved numerically, and the velocity and temperature profiles are obtained for various dimensionless numbers.

Copyright ( 2009 Mehrdad Massoudi et al. This is an open access article distributed under the Creative Commons Attribution License, which permits unrestricted use, distribution, and reproduction in any medium, provided the original work is properly cited.

\section{Introduction}

Combined heat transfer processes, such as convection-radiation, play a significant role in many chemical processes involving combustion, drying, fluidization, MHD flows, and so forth (see $[1,2])$. In general, the radiative process either occurs at the boundaries or as a term in the energy equation. The latter case is usually accomplished by a suggestion due to Rosseland (see [3]) where the radiative term is approximated as a flux in such a way that the term corresponding to radiation in the heat transfer (energy) equation now appears as a gradient term similar to Fourier's conduction term. This method has found much favor among many researchers especially those working with viscoelastic fluids and MHD flows (see [4-10]). Alternatively, radiation effects can be incorporated at the boundaries through appropriate (constitutive) assumption, such as the Stefan-Boltzmann condition. Free surface flows present a challenging problem to engineers as the combined convection-radiation at the boundaries has major applications in many industries.

Flow down an inclined plane occurs naturally as in the cases of avalanches and mudslides; it is also used for transporting and drying of bulk solids (such as agricultural 
and pharmaceutical products). It is a viscometric flow (see [11]) and one which amends itself to fundamental theoretical and experimental studies. Studies have shown that if the effects of viscous dissipation are included, strong nonlinearities in the temperate profiles are observed (see [12]). There are many cases in polymer rheology and lubrication, for example, where viscous dissipation cannot be ignored (see [13-15]). An important combustion-related issue is the effectiveness of handling and injecting of many non-Newtonian fluids such as coal-water slurries or coal-oil slurries. It has been demonstrated (see [16]) that substantial performance benefits can be obtained if the coal-water mixture is preheated. The effects of temperature change on coal slurry properties are controlled to a great extent by changes in the properties of the fluid. The constitutive equation used, most often, in these studies is the power law model. Although the power law model adequately fits the shear stress and shear rate measurements for many non-Newtonian fluids, it cannot always be used to accurately predict the pressure loss data measured during transport of a coal-liquid mixture in a fuel delivery system (see [17]).

Fluids of differential type (especially second and third grade fluids) have been studied extensively. For example, Rajagopal and Na [18] analyzed the natural convection of a thirdgrade fluid between two infinite parallel vertical plates; Szeri and Rajagopal [14] studied the flow of a third-grade fluid between heated parallel plates, and Massoudi and Christie $[19,20]$ considered the natural and forced convection of a third-grade fluid in an annulus. One of the recent advances in the theoretical studies in rheology is the development of generalized differential grade models. The simplicity of the form and the fact that these modified constitutive relations can be used to study shear-thinning/thickening, the decrease/increase in viscosity with increasing/decreasing shear rate, as well as predicting normal stress differences have opened the way for the solution to a series of engineering problems (see [21-25]).

In this work, we study the behavior of coal slurries, assuming that they can be represented by a constitutive equation similar to that of a generalized second-grade fluid, where the viscosity is also a function of temperature. Specifically, we revisit the problem considered by Massoudi and Phuoc [24] who studied the flow of a generalized secondgrade fluid down an inclined plane subject to convective heat transfer condition at the free surface, and consider the radiation boundary instead. The free surface is exposed to a high temperature and as a result a radiation boundary condition is used. The effects of viscous dissipation are also included in the heat transfer equation. The fully developed flow condition simplifies the problem and we obtain a set of three nonlinear ordinary differential equations which would have to be solved numerically. The equations are made dimensionless and the effects of different dimensionless numbers are discussed.

\section{Governing Equations}

The governing equations of motion are the conservation of mass, linear momentum, and energy equation. These are (see [26]) as follows.

Conservation of mass:

$$
\frac{\partial \rho}{\partial t}+\operatorname{div}(\rho \mathbf{u})=0
$$

where $\rho$ is the density of the fluid, $\partial / \partial t$ is the partial derivative with respect to time, and $\mathbf{u}$ is the velocity vector. For an isochoric motion we have $\operatorname{div} \mathbf{u}=0$. 
Conservation of linear momentum:

$$
\rho \frac{d \mathbf{u}}{d t}=\operatorname{div} \mathbf{T}+\rho \mathbf{b}
$$

where $\mathbf{b}$ is the body force vector, $\mathbf{T}$ is the stress tensor, and $d / d t$ is the total time derivative, given by $d(\cdot) / d t=\partial(\cdot) / \partial t+[\operatorname{grad}(\cdot)] \mathbf{u}$.

Conservation of energy:

$$
\rho \frac{d e}{d t}=\mathbf{T} \cdot \mathbf{L}-\operatorname{div} \mathbf{q}+\rho r
$$

where $e$ is the specific internal energy, $\mathrm{L}$ is the gradient of velocity, $\mathbf{q}$ is the heat flux vector, and $r$ is the radiant heating. Thermodynamical considerations require the application of the second law of thermodynamics or the entropy inequality. The various forms of this law are known as the Clausius-Duhem inequality, or the principle of dissipation [27, page 295] which states that

$$
\frac{1}{\rho} \operatorname{tr}(\mathrm{TD})+\theta \dot{\eta}-\dot{e} \geq 0
$$

where $\theta$ is the temperature field, $\mathbf{D}$ is the symmetric part of the velocity gradient, and $\eta$ is the specific entropy. We will not consider the constraints imposed due to Clausius-Duhem inequality (entropy law). In order to "close" these equations, we need to provide constitutive relations for $\mathbf{T}, \mathbf{q}$, and $r$. The application of the second law to radiative heat processes is by no means an easy and straightforward matter (see [28]) as assumed or implied in books and research articles. In this paper, we use the thermodynamical results of Dunn and Fosdick [29] without applying (2.4) directly to our constitutive relation.

\section{Constitutive Relations}

Perhaps the simplest model which can describe the normal stress effects (which could lead to phenomena such as "die-swell", and "rod-climbing" which are manifestations of the stresses that develop orthogonal to planes of shear) is the second-grade fluid, or the Rivlin-Ericksen fluid of grade two (see $[27,30]$ ). This model has also been used and studied extensively and is a special case of fluids of differential type. For a second-grade fluid the stress is given by

$$
\mathbf{T}=-p \mathbf{1}+\mu \mathbf{A}_{1}+\alpha_{1} \mathbf{A}_{2}+\alpha_{2} \mathbf{A}_{1}^{2}
$$

where $p$ is the indeterminate part of the stress due to the constraint of incompressibility, $\mu$ is the coefficient of viscosity, $\alpha_{1}$ and $\alpha_{2}$ are material moduli which are commonly referred to as the normal stress coefficients. The kinematical tensors $\mathbf{A}_{1}$ and $\mathbf{A}_{2}$ are defined through $\mathbf{A}_{1}=\mathbf{L}+\mathbf{L}^{T}, \mathbf{A}_{2}=\left(d \mathbf{A}_{1} / d t\right)+\mathbf{A}_{1} \mathbf{L}+(\mathbf{L})^{T} \mathbf{A}_{1}$, and $\mathbf{L}=\operatorname{grad} \mathbf{u}$. The thermodynamics and stability of fluids of second grade have been studied in detail by Dunn and Fosdick [29]. They show that if the fluid is to be thermodynamically consistent in the sense that all motions of the fluid 
meet the Clausius-Duhem inequality and that the specific Helmholtz free energy of the fluid be a minimum in equilibrium, then

$$
\begin{gathered}
\mu \geq 0, \\
\alpha_{1} \geqq 0, \\
\alpha_{1}+\alpha_{2}=0 .
\end{gathered}
$$

It is known that for many non-Newtonian fluids which are assumed to obey (3.1), the experimental values reported for $\alpha_{1}$ and $\alpha_{2}$ do not satisfy the restriction (3.2) 2,3 . In an important paper, Fosdick and Rajagopal [31] show that irrespective of whether $\alpha_{1}+\alpha_{2}$ is positive, the fluid is unsuitable if $\alpha_{1}$ is negative. In particular, they showed that if it is assumed that

$$
\mu>0, \quad \alpha_{1}<0, \quad \alpha_{1}+\alpha_{2} \neq 0,
$$

which as many experiments have reported to be the case "for those fluids which the experimentalists assume to be constitutively determined by (3.1), at least sufficiently well as a second-order approximation" [31, page 147], then certain anomalous results follow. Fosdick and Rajagopal [31] proved a theorem which indicates that if $(3.3)_{2,3}$ hold, then an unusual behavioral property not to be expected for any rheological fluid occurs, namely, the larger the viscosity, keeping everything else fixed, the faster that initial data is amplified in motions which take place under strict isolation. For further details on this and other relevant issues in fluids of differential type, we refer the reader to the review article by Dunn and Rajagopal [32]. In recent years, Rajagopal and colleagues (see, e.g., [33, 34]) have devised a thermodynamic framework, the Multiple Natural Configuration Theory, by appealing to the maximization of the rate of entropy production to obtain a class of constitutive relations for many different types of materials. Unlike the traditional thermodynamic approach (e.g., [29]) whereby a form for the stress is assumed (or derived) and restrictions on the material parameters are obtained by invoking the Clausius-Duhem inequality, in this new thermodynamic framework, they assume specific forms for the Helmholtz potential and the rate of dissipation-reflecting on how the energy is stored in the body and the way in which the body dissipates it. In a recent study, Rajagopal and Srinivasa [35] have modified their theory to obtain various forms for the fluid models of differential type, specially the second grade fluid; interestingly, they also arrive at the same conclusion about the restrictions imposed on $\alpha_{1}$ and $\alpha_{2}$, namely that $\alpha_{1}+\alpha_{2}=0$.

In an effort to obtain a model that does exhibit both normal stress effects and shearthinning/thickening, Man and Sun [36] and Man [21] modified the constitutive equation for a second-grade fluid by allowing the viscosity coefficient to depend upon the rate of deformation. Their model was later generalized by Gupta and Massoudi [22, 37] by allowing the shear viscosity to also be a function of temperature. That is,

$$
\mathbf{T}=-p \mathbf{1}+\mu(\theta) \Pi^{m / 2} \mathbf{A}_{\mathbf{1}}+\alpha_{1} \mathbf{A}_{2}+\alpha_{2} \mathbf{A}_{1}^{2}
$$




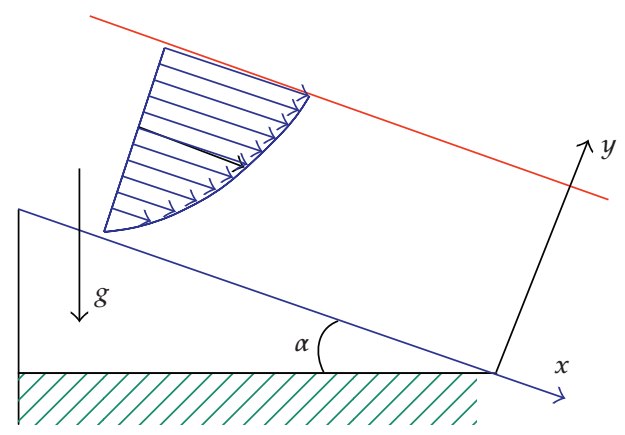

Figure 1

where $\Pi=(1 / 2) \operatorname{tr} \mathbf{A}_{1}^{2}$ is the second invariant of the symmetric part of the velocity gradient, $m$ is a material parameter, and $\mu(\theta)$ was assumed to obey the Reynolds viscosity model,

$$
\mu(\theta)=\mu_{0} \mathrm{e}^{-\mathrm{M} \theta},
$$

where

$$
M=n\left(\theta_{2}-\theta_{1}\right),
$$

when $m<0$, the fluid is shear-thinning, and if $m>0$, the fluid is shear-thickening. This model can find applications in many processes where preheating of the fuel is used as a means to enhance heat transfer effects. In addition, for many fluids such as lubricants, polymers, and coal slurries where viscous dissipation is substantial, an appropriate constitutive relation where viscosity is a function of temperature should be used. In this paper, we will use (3.4) (see [38] for a recent review). It also needs to be mentioned that second-grade fluids (or higher order models) raise the order of differential equations by introducing higherorder derivates into the equations. As a result, in general, one needs additional boundary conditions; for a discussion of this issue, see Rajagopal [39] and Rajagopal and Kaloni [40]. where

The constitutive relation for the heat flux vector is given by Fourier's assumption

$$
\mathbf{q}=-K \nabla \theta
$$

where $K$ is the thermal conductivity, and is assumed to be constant in this paper. The effects of radiation are assumed to be at the free surface and therefore will be discussed in the next section along with other boundary conditions.

\section{Flow Down an Inclined Plane}

Whenever nonlinear constitutive relations are studied, the solution procedures, for solving the governing equations, whether analytical or computational, are more complicated. Exact 


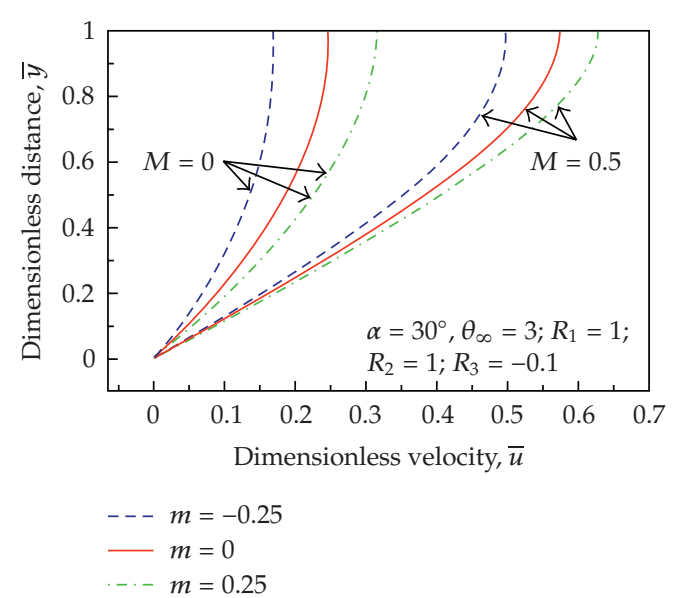

(a)

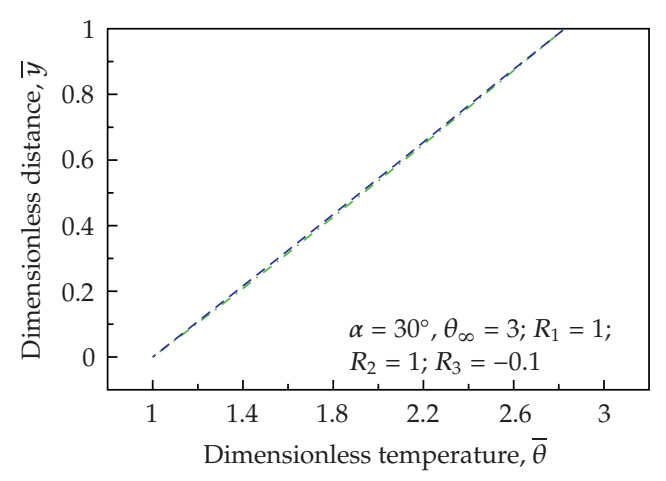

(c)

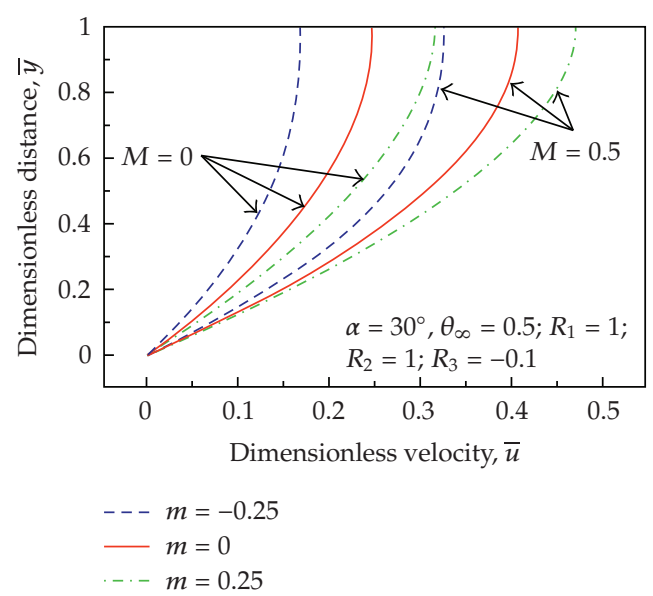

(b)

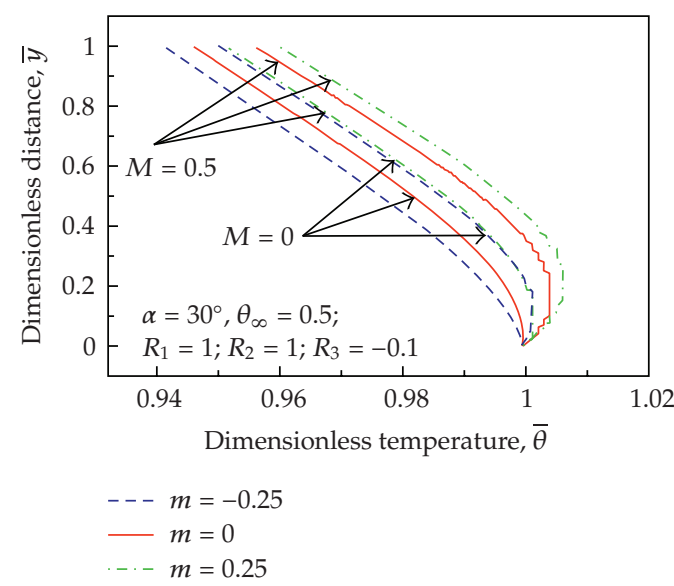

(d)

Figure 2: Velocity and temperature profiles.

solutions are very rare in heat transfer studies of nonlinear materials or multiphase flows. Next to the exact solutions, finding solutions to simple boundary value problems is extremely desirable. Most of the constitutive relations used in mechanics, whether non-Newtonian models, turbulence models, and so forth, when substituted in the general governing equations, that is, the balance laws, would produce a system of partial differential equations which at times are impossible to solve completely with the numerical techniques currently available. Therefore, from a modeling point of view, it is worthwhile to study problems where due to simplification of the kinematics of the flow or the boundary conditions, one obtains a system of (nonlinear) ordinary differential equations. The solution to these simpler problems would be useful for at least two different reasons: (i) They provide insight into the nature of these nonlinear constitutive relations, and (ii) they provide cases where the accuracy or convergence of solutions to the general multidimensional equations can be tested. Other interesting phenomena such as stability and uniqueness of solutions also sometimes arise. 


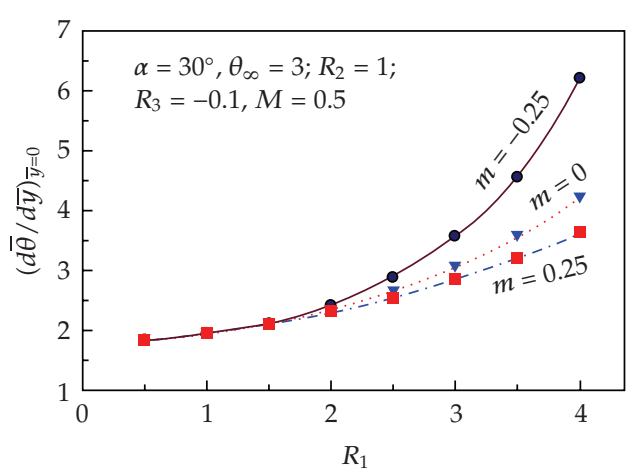

(a)

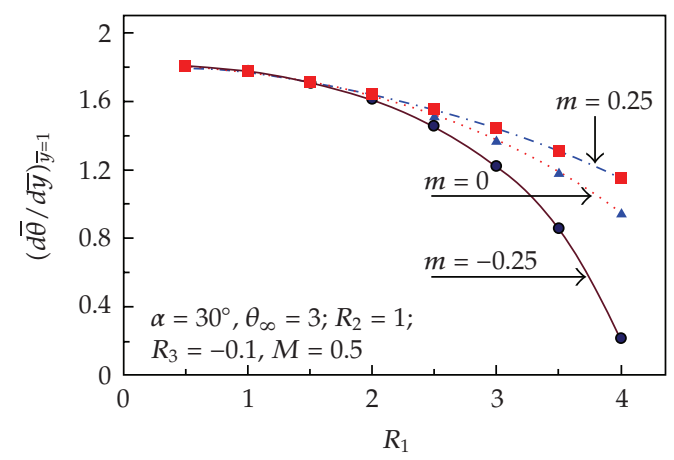

(c)

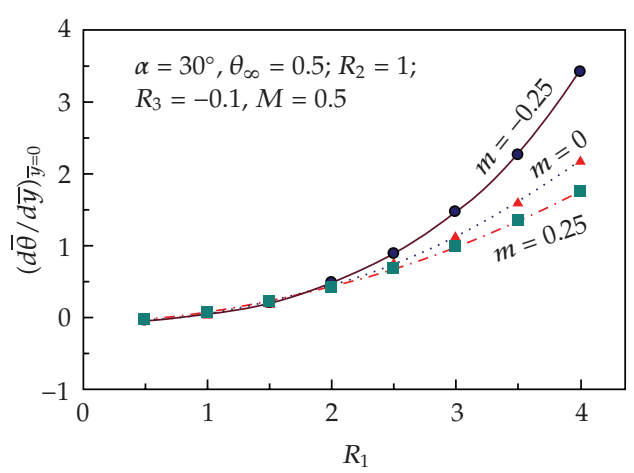

(b)

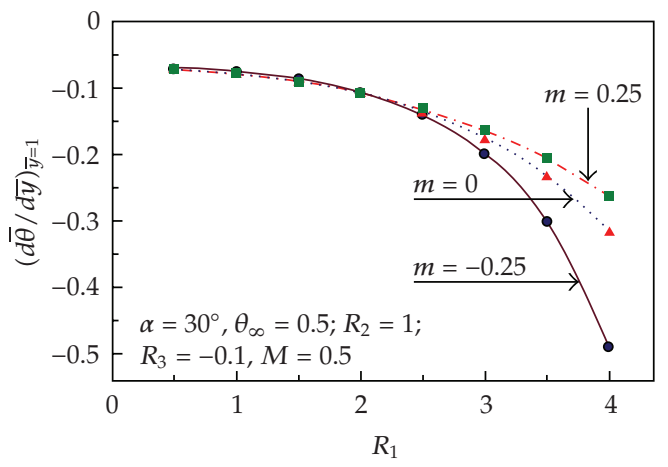

(d)

Figure 3: Heat transfer from the free surface: effect of $R_{1}$.

For the problem under consideration, we make the following assumptions:

(i) the motion is steady and fully developed,

(ii) the effect of radiant heating $r$ is included at the free surface,

(iii) the constitutive equation for the stress tensor is given by (3.4) and the constitutive equation for the heat flux vector is that of Fourier's assumption,

(iv) the velocity and temperature fields are of the form:

$$
\begin{gathered}
\mathbf{u}=u(y) \mathbf{i}, \\
\theta=\theta(y),
\end{gathered}
$$

where $y$ is the direction normal to the inclined plane, and $\mathbf{i}$ designates a unit vector in the direction of the flow (see Figure 1). The free surface is exposed to high ambient temperature and as a result a modified Stefan-Boltzmann correlation for radiation is used at that surface (see [41, page 331]). 


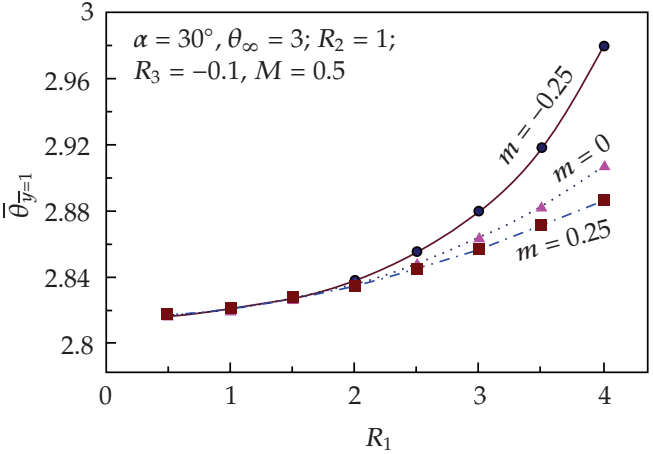

(a)

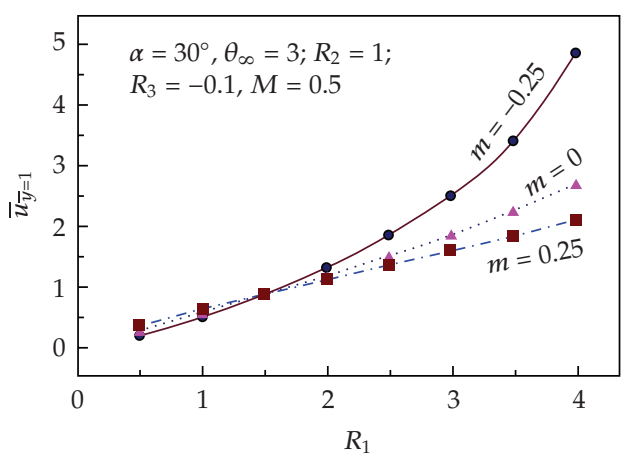

(c)

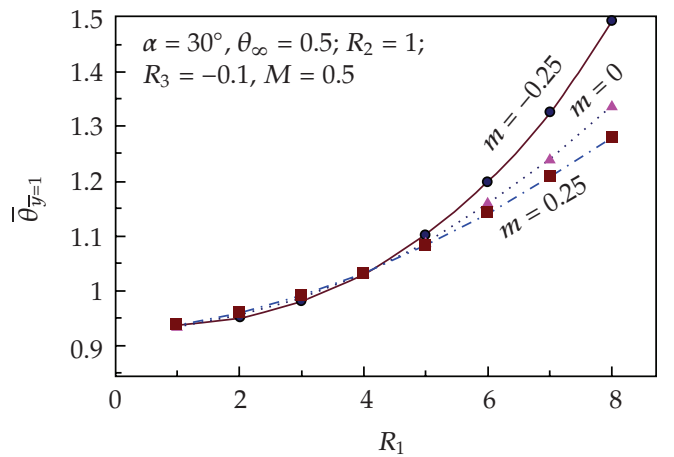

(b)

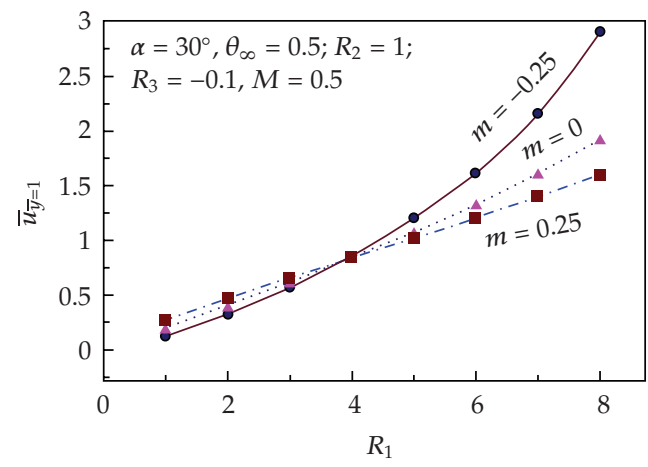

(d)

Figure 4: Free surface temperature and velocity as a function of $R_{1}$.

With the above assumptions, the conservation of mass is automatically satisfied and the momentum equation (2.2) in its dimensionless form becomes (see [24, 42] for details), having used the Reynolds viscosity model and integrating once

$$
\frac{d \bar{u}}{d \bar{y}}=R_{1}{ }^{1 /(m+1)}\left(e^{M \bar{\theta}}(1-\bar{y})\right)^{1 /(m+1)}(\sin \alpha)^{1 /(m+1)} .
$$

The energy equation (2.3) reduces to

$$
\frac{d^{2} \bar{\theta}}{d \bar{y}^{2}}+R_{2} \frac{1}{e^{M \bar{\theta}}}\left(\frac{d \bar{u}}{d \bar{y}}\right)^{m+2}=0
$$

where the dimensionless distance $\bar{y}$, the velocity $\bar{u}$, and the temperature $\bar{\theta}$ are assumed to be given by the following equations (see [43]):

$$
\bar{y}=\frac{y}{h} ; \quad \bar{u}=\frac{u}{V} ; \quad \bar{\theta}=\frac{\theta}{\theta_{w}} \quad \mu^{*}=\frac{\mu}{\mu_{o}}
$$


where $V$ is a reference velocity, $\theta_{w}$ is the wall temperature, and $h$ is the constant height to the free surface. The dimensionless numbers $R_{1}$ and $R_{2}$ are

$$
\begin{gathered}
R_{1}=\frac{\rho g h^{m+2}}{V^{m+1} \mu_{0}}, \\
R_{2}=\mu_{o}\left(\frac{V}{h}\right)^{m+2} \frac{h^{2}}{\theta_{w} K^{\prime}}
\end{gathered}
$$

Now, $R_{1}$ is the ratio of the viscous effects to the gravitational effects

$$
\mathrm{R}_{1}=\frac{\rho \mathrm{g} h^{m+2}}{\mathrm{~V}^{m+1} \mu_{0}}=\frac{\mathrm{g} h}{V^{2}}\left(\frac{\rho h^{m+1} V^{1-m}}{\mu_{0}}\right)=\frac{\mathrm{Re}}{(\mathrm{Fr})^{2}}
$$

related to the Reynolds and Froude numbers, and $R_{2}$ is a measure of viscous dissipation

$$
\mathrm{R}_{2}=\mu_{\mathrm{o}}\left(\frac{V}{h}\right)^{m+2} \frac{h^{2}}{\theta_{w} K}=\left(\left(\frac{c_{p} \mu}{K}\right)\left(\frac{V}{h}\right)^{m}\right)\left(\frac{V^{2}}{c_{p} \theta_{w}}\right)=\operatorname{Pr} \mathrm{Ec},
$$

related to the Prandtl and Eckert numbers. At the wall of the inclined we assume the noslip condition for the velocity and a constant temperature $\theta_{w}$. At the free surface, the notraction boundary condition is imposed on the stress tensor $\left(\left.t_{x}\right|_{y=h}=T_{x x} n_{x}+T_{x y} n_{y}+T_{x z} n_{z}=\right.$ $\mu\left[\left|u^{\prime}\right|^{2}\right]^{m / 2} u^{\prime}=0$ and $\left.t_{y}\right|_{y=h}=T_{y x} n_{x}+T_{y y} n_{y}+T_{y z} n_{z}=-p+\left(2 \alpha_{1}+\alpha_{2}\right) u^{\prime 2}=0$, where by defining a modified pressure $p^{*}=p\left(2 \alpha_{1}+\alpha_{2}\right) u^{\prime 2}$, the second relationship implies that $p^{*}=0$ at $\left.y=h\right)$, (see [24]), and for the temperature we apply the Stefan-Boltzmann condition (see also [44])

$$
q=\varepsilon \sigma\left(\theta_{h}^{4}-\theta_{\infty}^{4}\right)
$$

where the surrounding temperature is designated as $\theta_{\infty}$ and the temperature at the free surface is $\theta_{h}$. (The importance of radiation boundary condition for composite plates is discussed by Miller and Weaver [45], for fluid-particle flow by Chamkha [46], and for packed beds by $\mathrm{Wu}$ and $\mathrm{Chu}$ [47].)The boundary conditions become

$$
\begin{gathered}
\text { at } \bar{y}=0:\left\{\begin{array}{l}
\bar{u}=0, \\
\bar{\theta}=1,
\end{array}\right. \\
\text { at } \bar{y}=1:\left\{\begin{array}{l}
\frac{d \bar{u}}{d \bar{y}}=0, \\
\frac{d \bar{\theta}}{d \bar{y}}=R_{3}\left(\bar{\theta}_{\bar{y}=1}^{4}-\bar{\theta}_{\infty}^{4}\right),
\end{array}\right.
\end{gathered}
$$

where

$$
R_{3}=-\frac{h \varepsilon \sigma \theta_{w}^{3}}{K}
$$




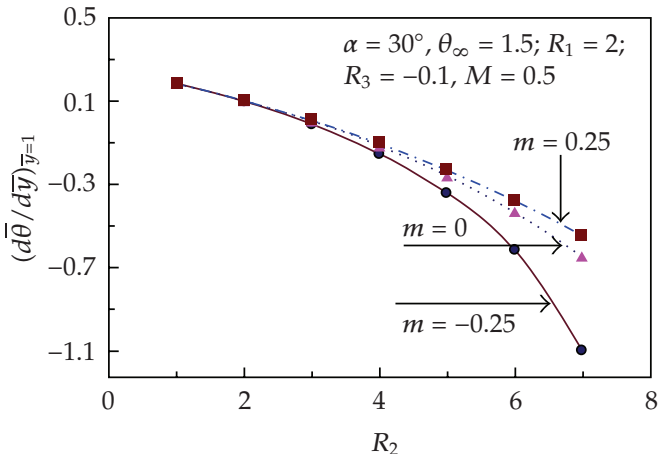

(a)

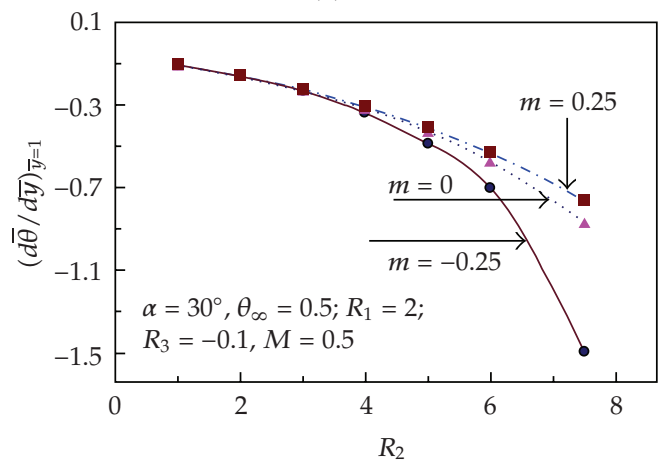

(c)

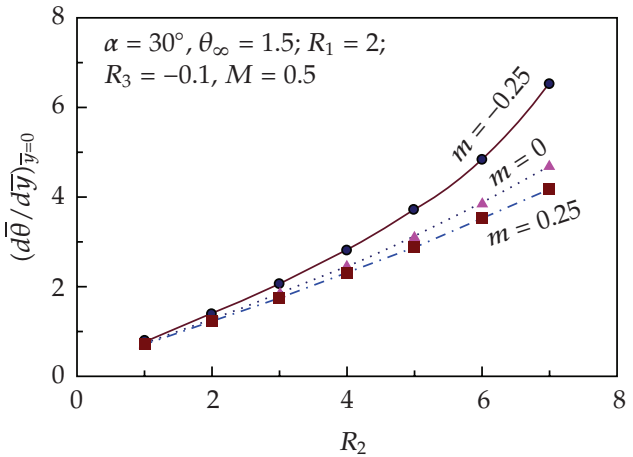

(b)

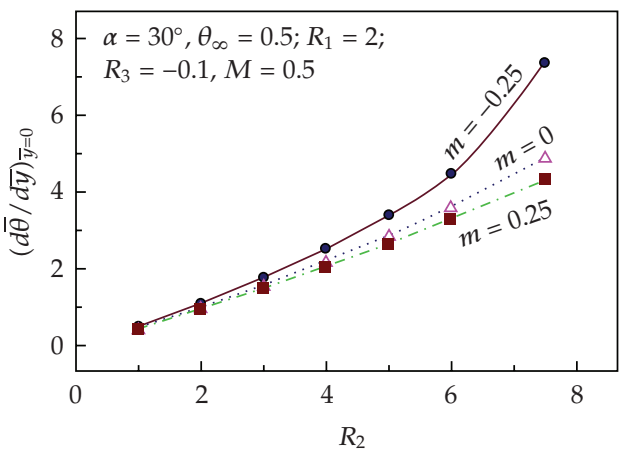

(d)

Figure 5: Heat transfer from the free surface: effect of $R_{2}$.

is a measure of the emissivity to the thermal conductivity and

$$
\bar{\theta}_{\infty}=\frac{\theta_{\infty}}{\theta_{w}} .
$$

It can be seen that the following material parameters $m, M$, the angle of inclination $\alpha$, a dimensionless temperature at infinity $\bar{\theta}_{\infty}$, and three dimensionless numbers $R_{1}, R_{2}$, and $R_{3}$ determine the flow and temperature patterns.

\section{Numerical Solutions}

Based on our previous experience we use the following values:

$$
\begin{aligned}
m & =-0.25,0,0.25, \\
M & =0,0.5,3.5, \\
\alpha & =30 \text { degrees, } \\
R_{1} & =0.5,2,4, \\
R_{2} & =1,5,10, \\
R_{3} & =-0.1, \\
\bar{\theta}_{\infty} & =0.5,1.5,3 .
\end{aligned}
$$


When the viscosity does not depend on the temperature, that is, $M=0$, the solutions are given analytically as

$$
\begin{gathered}
\bar{u}=\left(R_{1} \sin \alpha\right)^{1 /(m+1)} \frac{m+1}{m+2}\left[1-(1-\bar{y})^{(m+2) /(m+1)}\right] \\
\frac{d^{2} \bar{\theta}}{d \bar{y}^{2}}=-R_{2}\left(R_{1} \sin \alpha\right)^{(m+2) /(m+1)}(1-\bar{y})^{(m+2) /(m+1)} \\
\frac{d \bar{\theta}}{d \bar{y}}=R_{2}\left(R_{1} \sin \alpha\right)^{(m+2) /(m+1)} \frac{m+1}{2 m+3}(1-\bar{y})^{(2 m+3) /(m+1)}+R_{3}\left(\bar{\theta}_{\bar{y}=1}^{4}-\bar{\theta}_{\infty}^{4}\right) \\
\theta=R_{2}\left(R_{1} \sin \alpha\right)^{(m+2) /(m+1)} \frac{m+1}{2 m+3} \frac{m+1}{3 m+4}\left[1-(1-\bar{y})^{(3 m+4) /(m+1)}\right]+R_{3}\left(\bar{\theta}_{\bar{y}=1}-\bar{\theta}_{\infty}^{4}\right) y+1 .
\end{gathered}
$$

When $M \neq 0,(4.2)$ and (4.3) are coupled and they must be solved simultaneously. Following the numerical procedures reported by Massoudi and Phuoc [24], the dimensionless temperature and velocity are obtained for $m=-0.25,0.0$, and 0.25 by keeping $\alpha=30^{\circ}, R_{3}=$ -0.1 , and $M=0.5$ and using $\bar{\theta}_{\infty}, R_{1}$, and $R_{2}$ as parameters. Figure 2 shows the velocity distribution for both heating and cooling conditions for the case of temperature-dependent $(M=3.5)$ and temperature-independent $(M=0.0)$ viscosity. For the heating case we use $\bar{\theta}_{\infty}=3.5$ and for the cooling case $\bar{\theta}_{\infty}=0.5$. All other parameters are kept constant $(\alpha=30$, $\left.R_{1}=0.5 ; R_{2}=1 ; R_{3}=-0.1\right)$. The results are calculated for a shear-thinning fluid $(m=-0.25)$, the Newtonian fluid, $(m=0)$ and a shear-thickening fluid $(m=0.25)$. When $M=0$, the velocity does not depend on the temperature and therefore the distribution is the same for $\bar{\theta}_{\infty}=0.5$ and $\bar{\theta}_{\infty}=3$. In this case the temperature profile depends significantly on $\bar{\theta}_{\infty}$. In the cooling case where $\bar{\theta}_{\infty} \prec \bar{\theta}_{w}$ the temperature profiles are affected whether the fluid is shearthinning, Newtonian, or shear-thickening. When $\bar{\theta}_{\infty}>\bar{\theta}_{w}$, the effects of $m$ are minimal due to the viscosity decrease with higher temperature.

The effects of the dimensionless parameter $R_{1}$ on the heat transfer, the dimensionless temperature and velocity at the free surface are presented in Figures 3 and 4 . The calculations are made using $\bar{\theta}_{\infty}=3.0$ and 0.5 while all other parameters are kept constant. For the conditions used here, the heat transfer at the wall (the surface of the incline), as well as from the free surface is seen to increase with $R_{1}$. In the heating case, $\bar{\theta}_{\infty}=3.0$, the wall temperature is always lower than that of the surrounding, and it increases as $R_{1}$ increases. In the cooling case, $\bar{\theta}_{\infty}=0.5$, the free surface temperature is lower than the wall temperature when $R_{1}$ is less than about 1.75 indicating that the fluid is heated. For $R_{1}$ higher than this value, the free surface temperature is higher than both the wall temperature and the temperature of the surrounding. This is due to the fact that $R_{1}$ represents the effect of the gravity force such that an increase in $R_{1}$ leads to an increase the dimensionless velocity of the free surface as shown in Figure 4. As a result, the fluid is heated due to the kinetic energy that is converted into thermal energy.

The effects of $R_{2}$ on the heat transfer, temperature, and velocity at the free surface are shown in Figures 5 and 6 for the case where $\alpha=30^{\circ}, R_{1}=2, R_{3}=-0.1, M=0.5$, and $\bar{\theta}_{\infty}=1.5$ and 0.5. When $\bar{\theta}_{\infty}=0.5$ as $R_{2}$ increases, $(d \theta / d \bar{y})_{\bar{y}=1}$ is negative and it continues to decrease while $(d \theta / d \bar{y})_{\bar{y}=0}$ is positive and increases indicating that the maximum temperature is 


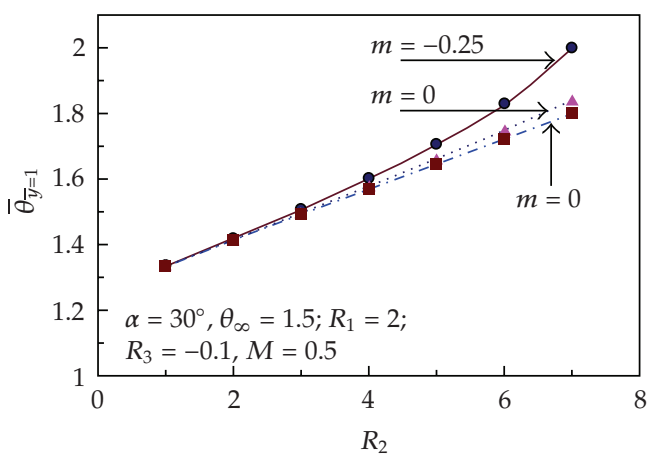

(a)

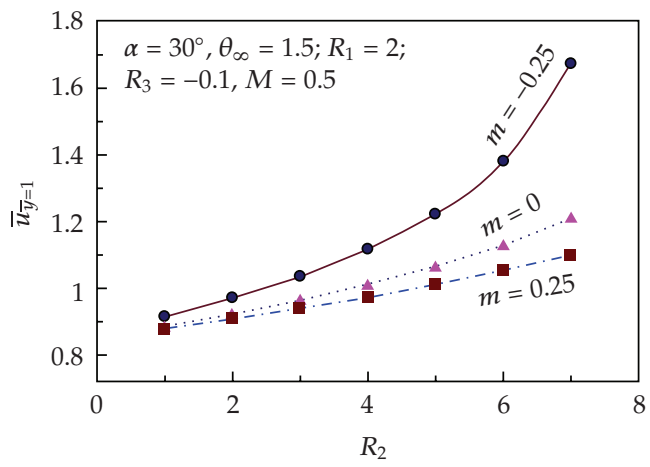

(c)

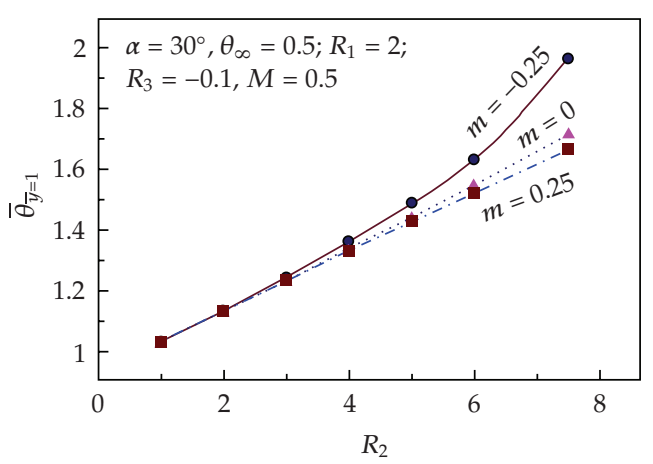

(b)

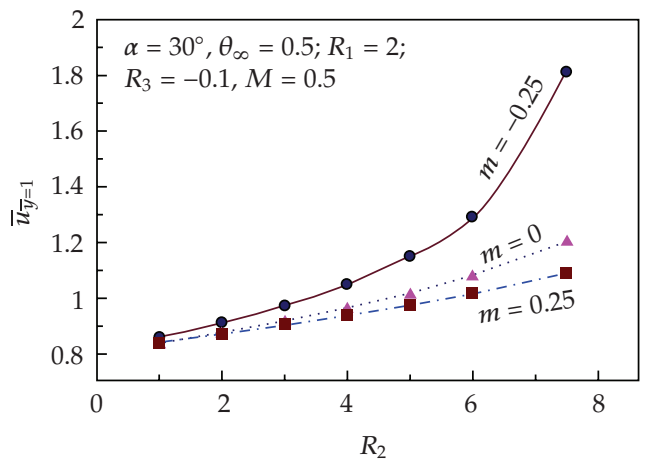

(d)

Figure 6: Free surface temperature and velocity as a function of $R_{2}$.

higher than both the wall (incline surface) temperature and the surrounding temperature; this maximum value is located somewhere in the flow field and heat is transferred from the fluid to both the wall and the free surface. When $\bar{\theta}_{\infty}=1.5$, as $R_{2}$ increases, $(d \theta / d \bar{y})_{\bar{y}=0}$ is positive and increases while $(d \theta / d \bar{y})_{\bar{y}=1}$ is positive and decreases when $R_{2}$ is less than 3.0. The free surface temperature is lower than that of the surrounding. Thus, in this range of $R_{2}$ the highest temperature is that of the surrounding and the fluid is heated. When $R_{2}$ is increased to a higher value than 3.0, $(d \theta / d \bar{y})_{\bar{y}=1}$ becomes negative and the free surface temperature is higher than that of the surrounding indicating that the fluid is heated (due to the effect of $R_{2}$ ) and the excess heat is transferred to both the wall and the surrounding. As shown by (4.3), the temperature is not only affected by the surrounding temperature but also by parameter $R_{2}$ which represents the effect of viscous dissipation. Increasing $R_{2}$ can lead to a situation that the viscous dissipation becomes a significant source in the energy equation.

\section{Conclusions}

In this paper we have studied the fully developed flow of a generalized second-grade fluid down an inclined plane. The viscosity is a function of the shear rate (shear-thinning or shear-thickening) and it also depends on the temperature in an exponential way. The effects of radiation boundary condition at the free surface are considered. The boundary value problem is solved numerically, and the velocity and temperature profiles are obtained for 
various dimensionless numbers. The following material parameters $m$ (power-law exponent accounting for shear-rate dependence of the viscosity), $M$ (an exponent accounting for temperature dependence of viscosity), the angle of inclination $\alpha$, a dimensionless temperature at infinity $\bar{\theta}_{\infty}=\theta_{\infty} / \theta_{w}$, and three dimensionless numbers $R_{1}, R_{2}$, and $R_{3}$, where $R_{1}$ is the ratio of the viscous effects to the gravitational effects (related to the Reynolds and Froude numbers), $R_{2}$ is a measure of viscous dissipation (related to the Prandtl and Eckert numbers), and $R_{3}$ is a measure of the emissivity to the thermal conductivity.

When $M=0$, the velocity does not depend on the temperature and, therefore, the distribution is the same for $\bar{\theta}_{\infty}=0.5$ and $\bar{\theta}_{\infty}=3$. In this case the temperature profile depends significantly on $\bar{\theta}_{\infty}$. In the cooling case where $\bar{\theta}_{\infty} \prec \bar{\theta}_{w}$, the temperature profiles are affected whether the fluid is shear-thinning, Newtonian, or shear-thickening. When $\bar{\theta}_{\infty}>\bar{\theta}_{w}$ the effects of $m$ are minimal. When $\bar{\theta}_{\infty}=0.5$ and 3.0, the heat transfer at the wall (surface of the incline) as well as from the free surface is seen to increase with $R_{1}$. In the heating case, $\bar{\theta}_{\infty}=3.0$, the wall temperature is always lower than that of the surrounding and it increases as $R_{1}$ increases. In the cooling case, $\bar{\theta}_{\infty}=0.5$, the free surface temperature is lower than the wall temperature when $R_{1}$ is less than about 1.75 indicating that the fluid is heated.

\section{References}

[1] Ya. B. Zel'dovich and Yu. P. Raizer, Physics of Shock Waves and High Temperature Hydrodynamic Phenomena, Dover, Mineola, NY, USA, 1967.

[2] G. C. Pomraning, The Equations of Radiative Hydrodynamics, Dover, Mineola, NY, USA, 1973.

[3] J.-F. Clouet, "The Rosseland approximation for radiative transfer problems in heterogeneous media," Journal of Quantitative Spectroscopy and Radiative Transfer, vol. 58, no. 1, pp. 33-43, 1997.

[4] A. Raptis, C. Perdikis, and H. S. Takhar, "Effect of thermal radiation on MHD flow," Applied Mathematics and Computation, vol. 153, no. 3, pp. 645-649, 2004.

[5] A. Raptis, "Radiation and viscoelastic flow," International Communications in Heat and Mass Transfer, vol. 26, no. 6, pp. 889-895, 1999.

[6] P. S. Datti, K. V. Prasad, M. S. Abel, and A. Joshi, "MHD visco-elastic fluid flow over a non-isothermal stretching sheet," International Journal of Engineering Science, vol. 42, no. 8-9, pp. 935-946, 2004.

[7] R. C. Bataller, "Radiation effects for the Blasius and Sakiadis flows with a convective surface boundary condition," Applied Mathematics and Computation, vol. 206, no. 2, pp. 832-840, 2008.

[8] R. C. Bataller, "Radiation effects in the Blasius flow," Applied Mathematics and Computation, vol. 198, no. 1, pp. 333-338, 2008.

[9] R. C. Bataller, "Effects of viscous dissipation and radiation on the thermal boundary layer over a nonlinearly stretching sheet," Physics Letters A, vol. 372, no. 5, pp. 631-636, 2008.

[10] R. C. Bataller, "Viscoelastic fluid flow and heat transfer over a stretching sheet under the effects of a non-uniform heat source, viscous dissipation and thermal radiation," International Journal of Heat and Mass Transfer, vol. 50, no. 15-16, pp. 3152-3162, 2007.

[11] C. Truesdell, "The meaning of viscometry in fluid mechanics," Annual Review of Fluid Mechanics, vol. 6, pp. 111-146, 1976.

[12] M. Massoudi and N. K. Anand, "A theoretical study of heat transfer to flowing granular materials," International Journal of Applied Mechanics and Engineering, vol. 9, pp. 383-398, 2004.

[13] H. H. Winter, "Viscous dissipation in shear flows of molten polymers," Advances in Heat Transfer, vol. 13 , p. 205, 1977.

[14] A. Z. Szeri and K. R. Rajagopal, "Flow of a non-Newtonian fluid between heated parallel plates," International Journal of Non-Linear Mechanics, vol. 20, no. 2, pp. 91-101, 1985.

[15] A. Z. Szeri, Fluid Film Lubrication, Cambridge University Press, Cambridge, Mass, USA, 1998.

[16] C. Y. Tsai, M. Novack, and G. Roffe, "Rheological and heat transfer characteristics of flowing coalwater mixtures," DOE Report DOE/MC/23255-2763, December 1988.

[17] J. M. Ekmann, D. J. Wildman, and J. L. S. Chen, "Laminar flow studies of highly loaded suspensions in horizontal pipes," in Proceedings of the 2nd International Symposium on Slurry Flows, vol. 38, pp. 85-92, ASME, FED, 1986. 
[18] K. R. Rajagopal and T. Y. Na, "Natural convection flow of a non-Newtonian fluid between two vertical flat plates," Acta Mechanica, vol. 54, no. 3-4, pp. 239-246, 1985.

[19] M. Massoudi and I. Christie, "Natural convection flow of a non-Newtonian fluid between two concentric vertical cylinders," Acta Mechanica, vol. 82, no. 1-2, pp. 11-19, 1990.

[20] M. Massoudi and I. Christie, "Effects of variable viscosity and viscous dissipation on the flow of a third grade fluid," International Journal of Non-Linear Mechanics, vol. 30, p. 687, 1995.

[21] C.-S. Man, "Nonsteady channel flow of ice as a modified second-order fluid with power-law viscosity," Archive for Rational Mechanics and Analysis, vol. 119, no. 1, pp. 35-57, 1992.

[22] G. Gupta and M. Massoudi, "Flow of a generalized second grade fluid between heated plates," Acta Mechanica, vol. 99, no. 1-4, pp. 21-33, 1993.

[23] M. Massoudi and T. X. Phuoc, "Flow of a generalized second grade non-Newtonian fluid with variable viscosity," Continuum Mechanics and Thermodynamics, vol. 16, no. 6, pp. 529-538, 2004.

[24] M. Massoudi and T. X. Phuoc, "Fully developed flow of a modified second grade fluid with temperature dependent viscosity," Acta Mechanica, vol. 150, no. 1-2, pp. 23-37, 2001.

[25] M. Massoudi, A. Vaidya, and R. Wulandana, "Natural convection flow of a generalized second grade fluid between two vertical walls," Nonlinear Analysis: Real World Applications, vol. 9, no. 1, pp. 80-93, 2008.

[26] J. C. Slattery, Advanced Transport Phenomena, Cambridge University Press, New York, NY, USA, 1999.

[27] C. Truesdell and W. Noll, The Non-Linear Field Theories of Mechanics, Springer, New York, NY, USA, 2nd edition, 1992.

[28] S. E. Wright, "The Clausius inequality corrected for heat transfer involving radiation," International Journal of Engineering Science, vol. 45, no. 12, pp. 1007-1016, 2007.

[29] J. E. Dunn and R. L. Fosdick, "Thermodynamics, stability, and boundedness of fluids of complexity 2 and fluids of second grade," Archive for Rational Mechanics and Analysis, vol. 56, no. 3, pp. 191-252, 1974.

[30] R. S. Rivlin and J. L. Ericksen, "Stress-deformation relations for isotropic materials," J. Ration. Mech. Anal., vol. 4, pp. 323-425, 1955.

[31] R. L. Fosdick and K. R. Rajagopal, "Anomalous features in the model of "second order fluids"," Archive for Rational Mechanics and Analysis, vol. 70, no. 2, pp. 145-152, 1979.

[32] J. E. Dunn and K. R. Rajagopal, "Fluids of differential type: critical review and thermodynamic analysis," International Journal of Engineering Science, vol. 33, no. 5, pp. 689-729, 1995.

[33] K. R. Rajagopal and A. R. Srinivasa, "A thermodynamic frame work for rate type fluid models," Journal of Non-Newtonian Fluid Mechanics, vol. 88, no. 3, pp. 207-227, 2000.

[34] K. R. Rajagopal and A. R. Srinivasa, "Modeling anisotropic fluids within the framework of bodies with multiple natural configurations," Journal of Non-Newtonian Fluid Mechanics, vol. 99, no. 2-3, pp. 109-124, 2001.

[35] K. R. Rajagopal and A. R. Srinivasa, "On the development of fluid models of the differential type within a new thermodynamic framework," Mechanics Research Communications, vol. 35, no. 7, pp. 483489, 2008.

[36] C. S. Man and Q. K. Sun, “On the significance of normal stress effects in the flow of glaciers," Journal of Glaciology, vol. 33, p. 268, 1987.

[37] G. Gupta and M. Massoudi, "Heat transfer and flow of a modified second grade fluid," in Procedings of the 28th National Heat Transfer Conference, vol. 174, pp. 7-12, ASME, HTD, Minneapolis, Minn, USA, July 1991.

[38] M. Massoudi and A. Vaidya, "On some generalizations of the second grade fluid model," Nonlinear Analysis: Real World Applications, vol. 9, no. 3, pp. 1169-1183, 2008.

[39] K. R. Rajagopal, "On boundary conditions for fluids of the differential type," in Navier-Stokes Equations and Related Nonlinear Problems, A. Sequeira, Ed., pp. 273-278, Plenum Press, New York, NY, USA, 1995.

[40] K. R. Rajagopal and P. N. Kaloni, "Some remarks on boundary conditions for flows of fluids of the differential type," in Continuum Mechanics and Its Applications, pp. 935-942, Hemisphere Press, New York, NY, USA, 1989.

[41] H. U. Fuchs, The Dynamics of Heat, Springer, New York, NY, USA, 1996.

[42] M. Massoudi and T. X. Phuoc, "Heat transfer in flowing granular materials: the effects of radiation boundary condition," International Journal of Applied Mechanics and Engineering, vol. 10, pp. 489-503, 2005.

[43] T. Y. Na, Computational Methods in Engineering Boundary Value Problems, vol. 145 of Mathematics in Science and Engineering, Academic Press, New York, NY, USA, 1979. 
[44] R. M. Saldanha da Gama, "On the conduction/radiation heat transfer problem in a body with wavelength-dependent properties," Applied Mathematical Modelling, vol. 28, no. 9, pp. 795-816, 2004.

[45] J. R. Miller and P. M. Weaver, "Temperature profiles in composite plates subject to time-dependent complex boundary conditions," Composite Structures, vol. 59, no. 2, pp. 267-278, 2003.

[46] A. J. Chamkha, "Effects of heat absorption and thermal radiation on heat transfer in a fluid-particle flow past a surface in the presence of a gravity field," International Journal of Thermal Sciences, vol. 39, no. 5, pp. 605-615, 2000.

[47] J.-W. Wu and H.-S. Chu, "Combined conduction and radiation heat transfer in plane-parallel packed beds with variable porosity," Journal of Quantitative Spectroscopy and Radiative Transfer, vol. 61, no. 4, pp. 443-452, 1999. 


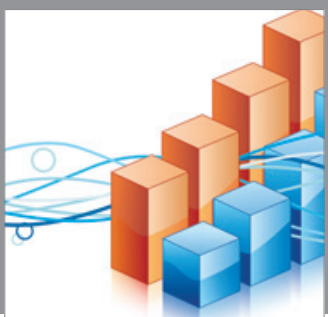

Advances in

Operations Research

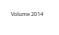

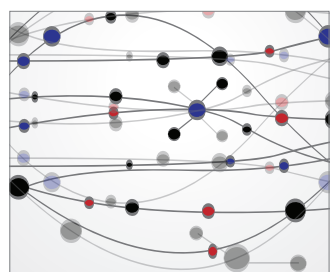

\section{The Scientific} World Journal
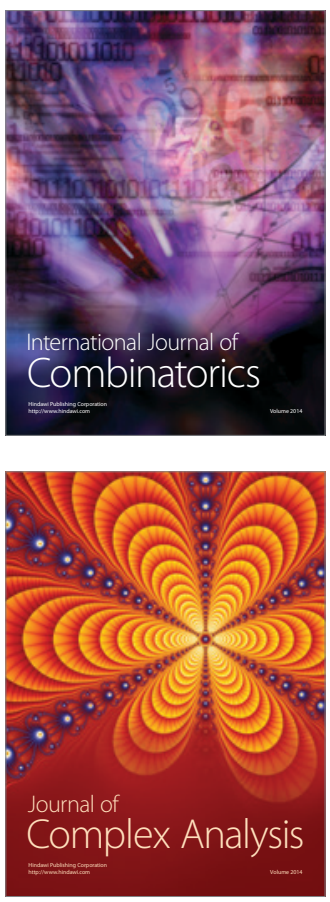

International Journal of

Mathematics and

Mathematical

Sciences
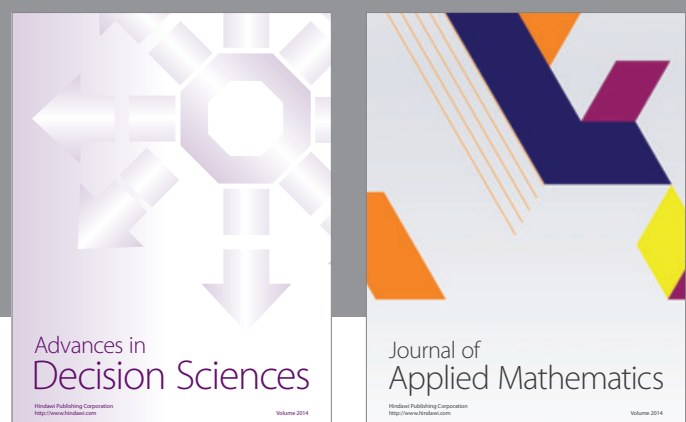

Journal of

Applied Mathematics
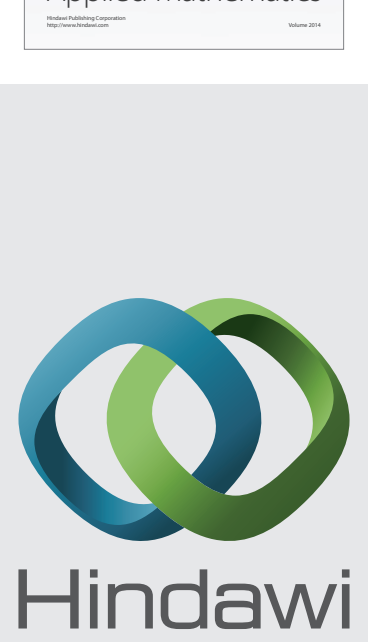

Submit your manuscripts at http://www.hindawi.com
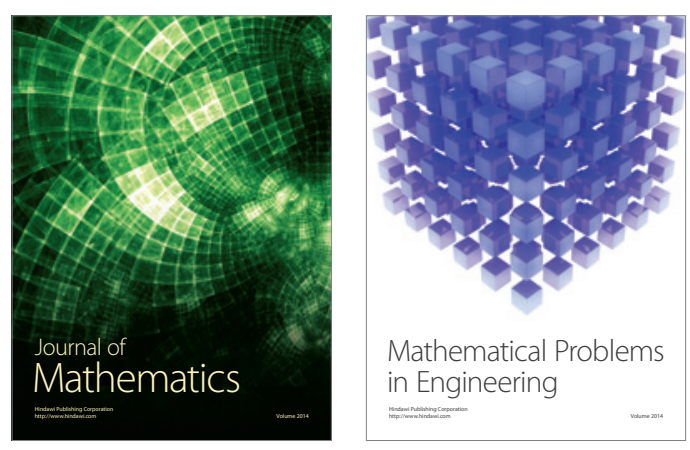

Mathematical Problems in Engineering
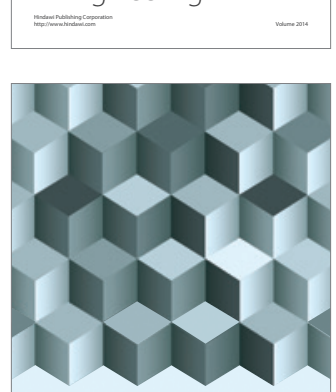

Journal of

Function Spaces
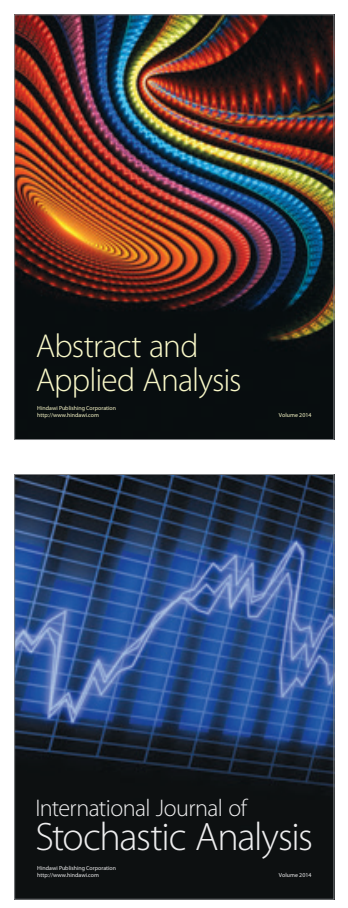

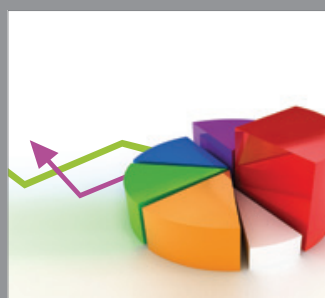

ournal of

Probability and Statistics

Promensencen
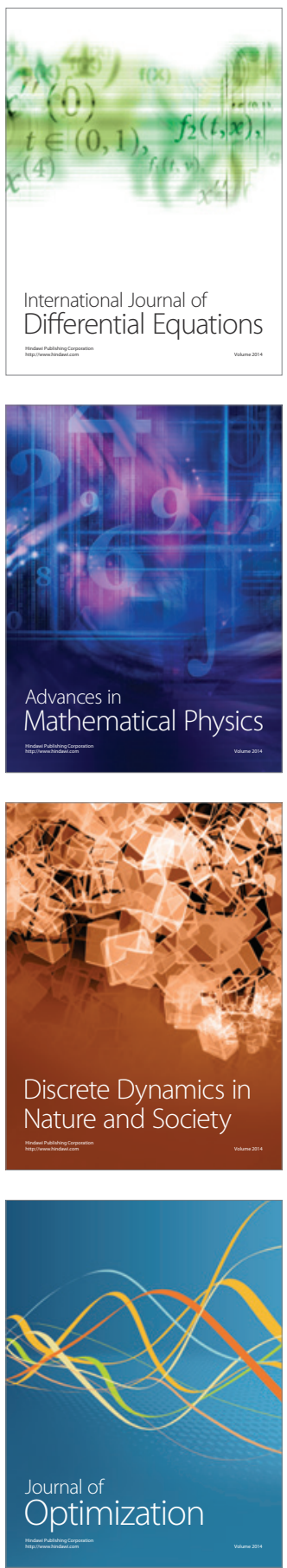